\title{
LA PROPUESTA DE LA UNIVERSIDAD NÓMADA: Una alternativa hacia la autoformación en la educación del arte
}

\author{
Carlos Moreno \\ Diana Boada \\ Tomás Bucheli \\ José Jiménez \\ Carolina Enríquez
}

\section{Resumen:}

Este artículo examina cómo a partir del proceso de reestructuración académica del 2009 se entablan paradigmas, que en el Seminario: Arte y Educación (CAC, 2012), hacen que se evidencie y divulgue la opinión de los estudiantes respecto a diversas problemáticas en torno a las ofertas académicas de las universidades, sus limitaciones y los procesos de profesionalización. Es entonces que se lleva a cabo la iniciativa: Universidad Nómada, creada y desarrollada por estudiantes. Además, se explican sus metodologías, posiciones frente a la academia, redes de trabajo, temáticas, contenidos y activación de procesos, en busca de generar apertura en áreas que carecen de atención por parte de instituciones de educación formal y discutir sobre el acceso a la educación. Se analiza el complejo social y la visibilización de lugares comunes y disímiles que ponen en crítica la idea de una cultura general, desde una red dinámica de trabajo teórico y práctico, conformada por múltiples microculturas, espacios, procesos e individuos fuera de la academia.

Palabras claves: Universidad - Experimental - Estudiantes - Pedagogía - Redes - Procesos - Arte

\begin{abstract}
:
This article examines how in 2009, educational paradigms are initiated based on the process of academic restructuration. The Art and Education Seminar (CAC 2012) evidenced students' point of view about difficulties on universities' academic programs and their limitations in professionalization processes. This is how this project starts: Nomadic University is a project created and developed by students. This article discusses the access to education of some humane groups as well as the methodologies, academic stances, networks, topics, contents, and activation of educational procedures, to generate broad mindedness in areas that lack attention from formal education institutions. This work analyzes social complexity and the difference in common and dissimilar places which challenge the idea of culture analyzing this concept through a theoretical dynamic network that involves multiple micro cultures and individuals outside academia.
\end{abstract}

Keywords: University, experimental, students, pedagogy, networks, processes, art

\section{Autores:}

La Asamblea de Estudiantes de Arte creada en el 2012, a raíz del Seminario: Arte y Educación; se define como un grupo que busca afianzar procesos de cooperación, constituyendo redes entre los participantes. Maneja prácticas pedagógicas alternativas de educación artística, para generar nuevos espacios para el aprendizaje. 
Cuando a la educación se le quedaron cortas las aulas

En septiembre de 2008 es expedido el Mandato Constituyente No. 14, que exige al CONEA (Consejo Nacional de Evaluación y Acreditación de la Educación Superior del Ecuador) la elaboración de un informe sobre el nivel de desempeño de los establecimientos de educación superior, con el fin de "garantizar su calidad, propiciando su depuración $\mathrm{y}$ mejoramiento" (CONEA, 2009) ${ }^{1}$. Por esta razón, las universidades del país tuvieron que someterse a un proceso de acreditación, así como de implementación de programas y renovaciones en las mallas curriculares en vista a mejorar la calidad de sus servicios educativos.

Para mayo del 2012, las universidades se encontraban en una fase posterior al rediseño curricular, acorde con la nueva Ley de Educación Superior, y los estudiantes tenían que enfrentarse a esta transición, en algunos casos sin participación activa en el diseño de las nuevas mallas. En este contexto se lleva a cabo el Seminario: Arte y Educación, en el Centro de Arte Contemporáneo de Quito, con la participación de estudiantes y docentes de las carreras de arte de las universidades del país, así como artistas, gestores culturales y colaboradores internacionales. Como resultado de la mesa de trabajo: ¿Cómo tejer procesos pedagógicos entre universidades, museos y comunidades?, los estudiantes que participaron en ella, proponen crear un espacio de autoformación, es decir un lugar que les permita gestionar su educación, que sea diseńado por y para estudiantes y mediante el cual puedan complementar los contenidos previstos en sus mallas curriculares.

1 Extraído de evaluación de desempeño institucional de las universidades y escuelas politécnicas del Ecuador. $(2009,11)$. CONAE
Se crea entonces la Asamblea de Estudiantes de Arte, con la premisa de convocar una red de iniciativas educativas, entendidos de forma integral y partiendo del hecho que cualquier espacio puede ser un lugar de aprendizaje, en donde se generan y crean vínculos a través de lo empírico, el conocimiento compartido y la autoformación, generando un modelo de educación activa, no directiva, colaborativa y autónoma, basándose en el arte como un criterio pedagógico y la pedagogía como un criterio artístico ${ }^{2}$.

Acorde con lo planteado por la Ley Orgánica de Educación Superior, en su artículo $17^{3}$, se incluye entre los fines de la educación superior encontrar una manera de integrarse dentro de la comunidad de manera crítica a través de la extensión de sus prácticas dentro de otro contexto, esto se planteó como una finalidad en el artículo 17 de la Ley Orgánica de Educación Superior.

Ases Arte plantea esta interacción, no a través de, sino con la comunidad universitaria dentro de varios contextos donde la práctica empírica de destrezas o aptitudes artísticas define al arte como metodología investigativa, comprendiendo que las dinámicas de participación dentro de estos entornos no académicos de enseñanza artística, son demarcados por la valoración del proceso a base de la calidad del entorno y las relaciones que pueden llegar a articular. Comprendiendo

2 Revisar de la ponencia de Luis Camnitzer en el foro PEDAGOGÍA RADICAL: el arte como educación, realizado en Panamá, en Mayo de 2012.

3 Artículo 17 de los programas y cursos de vinculación con la sociedad.- el reglamento de régimen académico normará lo relacionado con los programas y cursos de vinculación con la sociedad así como los cursos de educación continua, tomando en cuenta las características de la institución de educación superior, sus carreras y programas y las necesidades del desarrollo nacional, regional y local. 1 de septiembre del 2011. 
la extensión universitaria como espacios de distensión donde se busca complementar contenidos bipartitamente con actores sociales, académicos, y culturales, generando espacios de aprendizaje horizontales donde no se imparte o valida el conocimiento, se comparte y se desarrolla en prácticas colaborativas.

\section{Cuando asumimos las prácticas para nuestro propio aprendizaje}

Desde su formación y a través del tiempo, este colectivo ha definido de manera crítica e integradora su ideología, metodología y proyección. Como un espacio generador de procesos y experiencias educativas, a través de actividades gratuitas y de libre acceso, cuestionando los procesos institucionales en el arte, presentando propuestas que se articulan tanto en contenidos como en prácticas desde el conocimiento generado por el aprendizaje, pero enfocadas a un público abierto.

Reafirma su principio de autoformación a través de experiencias de aprendizaje, no de enseñanza, donde la educación libre o libertaria se diferencia de una pedagogía consciente. Freire en la pedagogía del oprimido resalta que:

La educación libertadora es incompatible con una pedagogía que, de manera consciente o mistificada, ha sido práctica de dominación. La práctica de la libertad solo encontrará adecuada expresión en una pedagogía en que el Oprimido tenga condiciones de descubrirse y conquistarse, reflexivamente, como sujeto de su propio destino histórico. (Freire, 1969, P. 6).
Es así que, al seguir la línea latinoamericana de pensamiento que autoafirma las prácticas locales, la Ases Arte parte de una evaluación alternativa que es activa, divergente y propositiva, fundamentada en que la experiencia de la persona que es partícipe de una actividad artística, puede generar productos que construyen y replantean referentes culturales en lo cotidiano, más allá de los criterios del aprendizaje, analizando las finalidades del espacio educativo y el fenómeno de las producciones independientes. Comprendiendo el cotidiano el comportamiento del individuo condicionado por el entorno :

El Habitus, un sistema de disposiciones funciona como esquema de clasificación orientando valoraciones, percepciones y acciones de los sujetos. es un conjunto de estructuras que representan la sociedad. (Bourdieu, 1995, p. 17)

Los planteamientos que propone Bourdieu dan cuenta de la automatización en que el individuo acepta su condición, papel o función dentro de los diferentes campos que estructuran lo social, aceptar las circunstancias e inscribirse dentro de un sistema funcional es una práctica que se reproduce desde los procesos educativos. Aun así, los espacios académicos del arte propician un enfoque crítico y constructivo a la sociedad y sus comunidades, y esto puede construir nuevas miradas y enfoques, pero tomando en cuenta que son pocos quienes estudian estas carreras nos deja la pregunta ¿Y los demás, que?

En el presente artículo se explica el proceso de la ASES Arte, el desarrollo de su proyecto $L a$ Universidad Nómada, así como su experiencia de trabajo en red. Se investiga de manera crítica el sistema educativo, los roles que plantea y las 
relaciones de poder que intervienen.

\section{Aprender lo que no se enseńa (temas, contenidos $\mathrm{y}$ prácticas transversales)}

La palabra "universidad" tiene una representación etimológica en la cual un todo diverso gira hacia un mismo fin. Guerra nos propone que esta debería estudiar la realidad conjunta:

La palabra Universidad como tal, significa etimológicamente hablando, "lo que está vuelto hacia lo uno", es decir, aquello que debe estudiar lo uno, sabiendo que lo uno, es el todo, el todo entero que nada excluye. Por lo tanto, la universidad debe estudiar todo, dicho con otras palabras, el objeto de conocimiento de la universidad es lo uno, el todo entero, toda la realidad o la Realidad en su conjunto. (Galindo Guerra, 2009, p.8).

Al proponer que todo gire a una misma finalidad se condiciona el proceso de aprendizaje a uno único que está caracterizado por su condición académica $^{4}$ (Bolonia, 1088). En el caso de las sociedades sudamericanas se han ignorado los fenómenos que se dan cuando un individuo se externaliza de su entorno referencial y aprende o asimila una gran cantidad de información que viene homologada desde las posturas que ha tomado el pensamiento en occidente. Es así, que el progreso del arte, o de cualquier otra rama que se imparte en las universidades funciona

$4 \quad$ Hernández, G., (2009). Origen de las universidades medievales en Italia (1 ed., Vol. 3, pp. 182-190). Bogotá, Colombia, Revista Educación y Desarrollo Social. Hernández, G., (2009). Origen de las universidades medievales en Italia (1 ed., Vol. 3, pp. 182-190). Bogotá, Colombia, Revista Educación y Desarrollo Social. sobre una serie de supuestos, que históricamente fundamentan la teoría y la práctica que deviene de ella, pero no se consideran los efectos que puede tener la reproducción de estos sistemas de conocimiento en la construcción de una cultura local, sobre estas condiciones de aprendizaje Foucault (1975) afirma:

\begin{abstract}
... cada alumno de acuerdo con su edad, sus adelantos y su conducta, ocupa ya un orden, ya otro (...) Entonces, la sala de clase formaría un gran cuadro único, de entradas múltiples, bajo la mirada cuidadosamente 'clasificadora' del maestro...
\end{abstract}

Foucault (1975, p.135.)

La propuesta experimental que maneja la Ases Arte, a través del proyecto Universidad Nómada, consiste en crear actividades en las cuales se pone en cuestión la finalidad del producto a generarse y así, a la vez, el desarrollo de un tema gira en torno a la importancia no solo de demostrar, sino también de gestar conocimiento entre todos.

Es así que se favorece la producción de espacios de encuentro interculturales con énfasis educativo ya que esta es una necesidad que surge desde la práctica artística:

la interculturalidad ha significado una lucha en la que han estado en permanente disputa asuntos como identificación cultural, derecho $\mathrm{y}$ diferencia, autonomía y nación. No es extraño que uno de los espacios centrales de esta lucha sea la educación, como institución política, social y cultural. Walsh ( 2009 p.5)

El enfoque que plantea la Universidad Nómada reside en mostrar la importancia de los procesos 
que están envueltos en la línea de producción de contenidos artísticos y culturales, valorando el nivel de incidencia que tieneel contexto sobrecada uno de estos aspectos que están marcados por una serie de comportamientos y situaciones sociales. Se propone una metodología de producción de valores artísticos y de aprendizaje, en su relación con espacios y momentos, que divergen de los procesos académicos y se convierten en el conocimiento que los participantes manejan y aplican en sus prácticas microculturales esta línea de acción configura nuevas maneras de participar López \& García en Microculturas y Cultura Organizacional: construcción dialéctica en la organización. afirman:

\section{... la cultura en la organización} no solo se configura por los aspectos de la estructura formal sino que existen otras dinámicas grupales espontáneas, distintas y a la vez, complementarias de los postulados que maneja la gestión de la cultura organizacional... Higuita López \& Leal García, Julieth C (2010, 150-167.)

De esta forma, los contenidos sobre los que se trabaja se construyen en el momento en que una actividad se concreta, proponiendo que las herramientas de la expresividad artística pueden funcionar como un eje transversal para atender problemáticas personales y colectivas.

\section{Educación sin fronteras (sobre la construcción de universidad)}

¿Qué se propone para la enseñanza? Se ha de buscar la construcción de una ciudadanía que logre al mismo tiempo ser crítica e integrada. Esto es, que sea parte del sistema político, que no se disocie de él, sino, por el contrario, lo conozca y tenga la capacidad de incidir en él. Al mismo tiempo, desde aspiraciones ciudadanas democráticas que desarrollen su evaluación sobre el mismo y demanden su mejora. Rodríguez ( p. 26 )

Después de un sondeo realizado en la FAUCE sobre las necesidades de los estudiantes, se generó el interés de responder a ellas desde una posición fuera de la experiencia académica, cuestionando el rol del estudiante como receptor pasivo de información, puesto que "en la escuela actual mientras más dócil sea un educando y más se deje llenar de narraciones de su educador, mejor educando será.” (Freire, 1970). Además de cuestionar el espacio académico como único lugar de aprendizaje, Ases arte parte de la idea de que se puede generar conocimiento en las relaciones interpersonales, proponiendo así la Universidad Nómada.

Dado que el arte se debate la mayoría de veces como el receptor o como el fin de un problema que se resuelve a partir de formulaciones estéticas, descartando aristas y problemáticas que el camino de la investigación sí debe considerar, por su alto nivel de retroalimentación en la comprobación del problema, es decir de su evaluación. Estos procesos de carácter integral, usualmente se ven truncados por el uso directivo de las metodologías educativas, o por la falta de participación de los asistentes, cuestiones que la construcción colectiva resuelve de manera activa dentro de procesos de equidad en el ambiente de trabajo donde se relaciona de mejor manera arte y calidad de vida:

Para el desarrollo de esta función social de las artes como generadora de cambio (aunque no de forma masiva 
e inmediata), la educación artística puede y debe ofrecer ámbitos de exploración, reflexión y compromiso, de manera individual y colectiva, que se proyectan en la búsqueda de una mayor calidad en la relación de arte y vida. Abad j (p.17)

La participación en la Universidad Nómada se da espontáneamente y no es definitiva, nace desde el ímpetu de resolver los problemas inmediatos en el aprendizaje del arte, a través de esfuerzos colectivos, ocupación de espacios públicos y generación de recursos alternativos, que permiten, no únicamente obtener información sobre el problema, sino generar respuestas de forma experimental.

Todo esto busca crear y expandir campos de conocimiento a través del arte como proceso educativo, para cuestionar de manera crítica su pertinencia académica y plantearla desde otras aproximaciones sobre todo en la manera que el entorno define nuestros recursos, así como por la constancia en el proceso, que genera un alcance, que a través de la investigación y la experimentación podría ser un eje vital de la educación.

\section{Tejiendo Redes (activación de espacios y economías alternas)}

...toda la tarea de educar solo es auténticamente humanista en la medida en que procure la integración del individuo a su realidad nacional, en la medida en que pierda el miedo a la libertad: en la medida en que pueda crear en el educando un proceso de recreación, de búsqueda, de independencia y, a la vez, de solidaridad (Freire, P 14. 2007)
Si se tiene en cuenta que aún dentro de una geografía delimitada, como es el caso de Quito, se desarrollan una amplia gama de grupos que tienen sus propias características y que actúan como células dentro de un gran organismo urbano, en algunos casos alejados de la educación artística, sobre todo en la periferia. La creación de redes integradas por personas, microcolectivos e instituciones, genera en la localidad nuevas formas de aprendizaje y propicia el terreno ideal para la colaboración, el diálogo y la mediación de las experiencias significativas que se puedan producir alrededor de los objetivos, en participación de cada una de sus partes.

Se plantea que el conocimiento se constituye como una macrored de saberes, opiniones y posiciones que se generan a través de experiencias de vida y de cómo, estas se dinamizan en las actividades del cotidiano, es cada una de ellas esencialmente distinta y única, pero que forman parte de un colectivo, donde todas son indispensables. Es así que se afronta la distinción de contenidos académicos que existe en la educación tradicional, al otorgarles un valor que considera si deben ser abordados o no en el aula y de qué manera hacerlo.

Este tipo de red se alimenta cuando su estructura permeable se construye en colectivo, a partir de necesidades personales o comunitarias, en la que las aproximaciones que se generen desde otros campos del conocimiento ampliarán el debate sobre los espacios en los que sus procesos y actividades se llevan a cabo.

La línea de acción de ASES Arte plantea una construcción colectiva, fomentando procesos de valoración en términos no económicos que llegan a ser sustentables para todos los que forman parte de la misma, al manejar una serie 
de recursos y bienes amplios, puesto que de manera aislada no se habrían podido llevar a cabo como procesos individuales.

Este esquema de trabajo permite construir un alcance real, sobre la manera en que se relaciona al público dentro de la escena cultural, proponiendo un sistema pedagógico, donde la interacción puede modificar la relación de una producción artística cuando esta se desarrolla, por tener una variedad de partes trabajando sobre su premisa.

Al ejercer prácticas culturales fuera de un acercamiento económico, se valora de otra manera la forma en la que se maneja y concibe la cultura y la educación, ya que nuestra intención es entablar una economía de recursos que nos permite expandir nuestras propuestas, llevándose a cabo todas a la par, gracias al carácter pedagógico con el cual trabajamos y el alcance cultural que se forma en el consenso.

\section{El Jam de Dibujo y Otras Artes "Esperando a Godo"}

Desde febrero del 2014, ASES Arte proyecta su trabajo a partir del "Jam de dibujo \& otras artes, Esperando a Godot" ", que es un proyecto adscrito a la Universidad Nómada, y ha sido realizado en diferentes lugares de la ciudad, así como diversas temáticas, con la intención de generar un público fuera de la mentalidad académica y técnica, que da a entender que un "buen dibujo" solo puede ser realizado por las pocas personas que tienen un estudio previo o "habilidad" para ello.

5 Lleva el nombre de la obra de teatro de Samuel Beckett, por el carácter de esperar que las cosas pasen y la idea de esperar al público que está siempre por venir. Se le denomina "Jam" a partir de las sesiones de Jazz que se realizaban en E.E.U.U., a principios de los años 30, por su formato de improvisación.
Es así que por medio de ejercicios experimentales y lúdicos, dentro de un espacio de ocio, distensión y disfrute, generado también como parte de las premisas para las propuestas, se propone la interacción activa entre los participantes y las herramientas. El público que asiste mantiene la construcción de las actividades a través de la cooperación y producción de lenguajes comunes en el cual se elaboran y comparten incontables productos visuales, accionando contenidos que giran en torno al arte desde cualquier disciplina.

El dibujo se convierte en una manera de generar nodos entre espacios y su relación con la vida y experiencia del que lo usa, además, si se tiene presente la idea de evaluar la enseñanza del dibujo más allá del aprendizaje académico tradicional, tratando que las valoraciones primero sean según la capacidad de generar como iniciativa propia un jam, a partir de una idea que se apoya desde los integrantes de la Asamblea, segundo por la capacidad de constancia y permanencia en el tiempo para poder vivir diferentes experiencias de dibujo y otras artes a lo largo del ańo; tercero por la capacidad de utilizar este espacio de encuentro para proponer y relacionar actividades que apoyen a la propuesta de utilizar la experiencia para generar proyectos independientes de arte y otros campos de conocimiento, y por último la amplitud de convocatoria y difusión generada a través de un nodo importante de la red, que es el aprovechamiento de los nuevos medios y redes sociales como recurso de liberación de contenidos y herramienta de registro, así como evidencia para la construcción del archivo del proceso del espacio de improvisación o jam.

La metodología de jam viene ejecutándose en la ciudad de Quito dentro de otros espacios y con otras perspectivas. Hace algún tiempo, tenemos por ejemplo el jam de dibujo en la cafetina 
producido por Alegría Mateljan que comparte con nosotros su experiencia con Esperando a Godot:

"Mi experiencia fue y es muy grata, he aprendido mucho de su forma de trabajar, gestión e interés en trabajar en pro del arte, de sostener procesos y construir colectivamente. Además mencionar que el uso de diversas metodologías pedagógicas en los "jams" que han propuesto generan las ganas de autoeducarse y de trabajar desde la autogestión.”

Partícipes de esta experiencia hemos articulado varias prácticas que logren potenciar a su vez en el jam, Eduardo Cando integrante de la banda ecuatoriana Curare relata su experiencia con el jam:

"Yo trabajaba en el CAC junto a Tomás y Pepe y bueno a partir de este encuentro conocí a otras personas más que en el proceso de la experiencia de los "jams" fue convirtiéndose en una plataforma colectiva de colectivos. Luego no se limitaba a encontrar personas, sino a encontrar colectivos y procesos en algún punto, se le puso el nombre de "Esperando a Godot: Jam de dibujo y otras artes" por la necesidad de identificar concretamente este espacio y por qué además en el camino al principio eran jams de dibujo, una práctica que tiene gran acogida en diferentes niveles de la escena pero la exploración y los encuentros llevaron a integrar más manifestaciones artísticas el proceso generado y sostenido por personas concretas me parece una experiencia valiosa de gestión de la cultura desde espacios no formales y no convencionales desde lo lúdico y lo crítico.”

Además estudiantes de carreras afines también dan cuenta de su proceso dentro de los jams de Dibujo, como Mayra Miranda y Tiani Samay, estudiantes de Diseño Gráfico en la Metro y la Carrera de Teatro de la UCE respectivamente, opinan que:

"Creo que como todo proyecto, empezar fue crucial y también una prueba de resistencia y esmero para que la propuesta coja su curso. La idea de crear un momento para dibujar y poder ver a más personas dibujando creo que es lo que más animaba los jams aparte de las temáticas. El Jam más sentido o más fuerte creo fue el de los "stickers", y el de las tizas, se pudo ver como todos trabajamos por un mismo objetivo, igual que en el jam de Stop Motion.

Creo podría describir mi experiencia como alentadora a parte de divertida, existen muy pocos espacios en los que colectivamente sin importar si conoces a todos o a nadie. Se puede trabajar y ver en ese mismo momento los resultados, también sentirse bien recibido, y que el lugar no sea siempre el mismo que daba dinamismo a las actividades, aparte de ser un aporte a los lugares donde se realizaron los jams.

Apoyar propuestas como estas creo que logra sacudirnos un poco la mente para darnos cuenta que las opciones existen y solo hay que hacerlas."

La última exposición del proceso en el Arte Actual de la FLACSO, da cuenta de la importancia y urgencia de este espacio en la escena de la cultura no solo quiteña, sino también nacional e internacional, supe que han tenido encuentros en Colombia. Eso es mi experiencia, sintiéndome vinculado al proceso de algunas formas y reconociendo el trabajo sostenido de personas concretas, que no las voy a nombrar porque sabemos quiénes son.” 


\section{A manera de conclusión}

La Universidad Nómada propone una manera experimental de abordar procesos, que se dan en la experiencia social urbana, es así, que crea nexos entre comunidades, actores culturales y estudiantes que empiezan a entender cómo se desenvuelve y funciona el arte. A su vez busca que docentes y dirigentes de las facultades de arte generen vínculos con los estudiantes, con la intención de elaborar líneas de investigación y proyección conjunta, manteniendo espacios de diálogo para conocer y debatir conceptos, alrededor de problemáticas actuales relacionadas con la educación y el arte.

Ahora, Ases Arte busca generar procesos de intercambio entre estudiantes de diferentes universidades con el fin de generar un reflujo del proceso que llevó a crear una visión, tal vez acertada o no, de como se ve a la educación artística desde la voz del estudiantado. Se generarán espacios de encuentro y diálogo con proyecciones de participación conjunta que se desarrollarán como mesas de trabajo. Ahora solo podemos esperar y ver cómo se pronuncian las nuevas promociones sobre los temas críticos que devienen de varios procesos.

Se debe considerar que el papel que mantiene la academia sirve como detonador de propuestas, donde el estudiante debe apropiarse de la experiencia de aprendizaje para que esta sea activa, coherente con su entorno e inmediata. La cohesión de la realidad y el conocimiento fuera del aula se desarrolla en procesos colaborativos, que al mantenerse en un trabajo independiente de los ejes académicos, son generadores de estrategias de resistencia y abordan otra idea de la rentabilidad de los micro-colectivos culturales.
Además se entuende que los procesos del arte son parte de un entretejido social cultural, aparece actualmente la necesidad de generar intercambios con diferentes esferas que comprendan otras prácticas culturales, la Ases Arte participa activamente en buscar afianzar este tejido social que se está articulando desde Minga: Movimiento por las Culturas a través de "Encuentros Semilla" provinciales donde se evidencian diferentes núcleos donde poder generar interculturalidad. Generando un conocimiento desde lo local, buscando contextualizar las prácticas de autorreferencialidad de diferentes comunidades que tienen la posibilidad de generar sus propias narrativas desdeloslenguajesdelarte, perocon una apreciación desligada de las visiones occidentales.

Entender que las nuevas generaciones exploran, experimentan y transitan en la construcción de la experiencia vital desde varias posiciones y hacia diferentes objetivos, esto se convierte en un factor importante para la creación y consolidación de un conocimiento que disputa la profundidad de su contenido frente a la imposición de modelos homogéneos. Es importante empoderar a estas nuevas generaciones de un mundo que está siempre en construcción.

\section{Bibliografía}

- Beuys, J., (1995). Joseph Beuys: cada hombre, un artista: conversaciones en Documenta 5-1972. Madrid, España. Editorial Visor.

- Bourdieu, P., (1995). Las reglas del arte. España. Anagrama Ediciones.

- Camnitzer, L., (Mayo de 2013) Arte y Pedagogía. Foro Pedagogía Radical: el arte como educación. Simposio llevado a cabo en la Ciudad 
de Panamá, Panamá.

- CONEA. (2009). Evaluación del desempeño institucional de las universidades y escuelas politécnicas del Ecuador. Quito, Ecuador: CONEA.

- Jiménez, L., Aguirre, I., y Pimentel, L. (2009). Educación artística, cultura y ciudadanía. Quito Ecuador: OEI - Fundación Santillana .( p. 26 )

- Foucault, M., (2002). Vigilary Castigar (1 ed.). Buenos Aires. Siglo XXI Editores.

- Freire, P., (2007). La Educación como práctica de la Libertad (53 ed.). Montevideo, Tierra Nueva. Siglo XXI Editores.

- Freire, P., (1969), Pedagogía del oprimido Chile, Santiago, Tierra Nueva.

- Hernández, G., (2009). Origen de las universidades medievales en Italia (1 ed.). Bogotá, Colombia, Revista Educación y Desarrollo Social.

- Walsh C., (Marzo del 2009) ponencia presentada en el Seminario "Interculturalidad y Educación Intercultural", organizado por el Instituto Internacional de Integración del Convenio Andrés Bello en La Paz, Bolivia.

-Galindo Guerra, H., (2009). Palabreando 1.1 [en línea]. Febrero 2015, de Universidad Pedagógica Nacional (México)

Disponible en:

http://www.educa.upn.mx/hemeroteca/ business-mainmenu-29/163-num-01/182palabreando (2009).

- Higuita, D. y Leal, J., (2010). Microculturas y Cultura Organizacional: Construcción Dialéctica en la Organización [en línea]. Febrero 2015, de Universidad Nacional de Colombia

Disponible en: http://www.scielo.org.co/ scielo.php?script=sci_arttext $\&$ pid $=$ S0121$68052010000100009 \& \operatorname{lng}=$ es\&tlng=es..\%20.

- Hernández, G., (2009). Origen de las universidades medievales en Italia [en línea]. Enero de 2014, de Revista Educación y Desarrollo Social

Disponible en: http://www.umng.edu.co/ documents/63968/80128/SeccionIII.articulo3. pdf 